\title{
Dietary Application for the Management of Patients with Hemodialysis: A Formative Development Study
}

\author{
Cosette Fakih El Khoury ${ }^{1}$, Mirey Karavetian ${ }^{2}$, Ruud J. G. Halfens ${ }^{1}$, Rik Crutzen ${ }^{2}$, Dayana El Chaar ${ }^{3}$, \\ Jos M. G. A. Schols ${ }^{1,4}$ \\ 'Department of Health Services Research, Care and Public Health Research Institute, Maastricht University, Maastricht, Netherlands \\ ${ }^{2}$ Department of Health Sciences, Zayed University, Dubai, UAE \\ ${ }^{3}$ Department of Natural Sciences, School of Arts and Science, Lebanese American University, Beirut, Lebanon \\ ${ }^{4}$ Department of Family Medicine, Faculty of Health, Medicine and Life Sciences, Maastricht University, Maastricht, Netherlands
}

Objectives: To describe the step-by-step person-centered, theory-based development of the KELA.AE app for Arabic speaking hemodialysis patients. Methods: A step-by-step person-driven theory-based approach was conducted to develop a self-monitoring and educational dietary app for hemodialysis patients. The development follows the Integration, Design, Assessment, and Sharing (IDEAS) framework. Qualitative, semi-structured interviews with 6 hemodialysis patients and 6 healthcare practitioners (dietitians and nephrologists) were performed to assess the need for an app, the willingness to use an app, and features desired in an app. Results: The KELA.AE app, which includes a self-monitoring feature, CKD-friendly recipes, and a theory-based, evidence-based educational feature was developed. Qualitative analysis of interviews revealed two predominant themes from patient interviews 'Experience with the diet', 'App evaluation', and one theme from interviews with healthcare practitioners 'App evaluation'. Patients expressed frustration with current accessibility of dietary information along with the need for educational materials in the app. The review of the KELA.AE prototype was positive overall, and patients reported a willingness to use the app. Healthcare practitioners considered the app accurate, simple, and culturally sensitive but expressed concerns about app misuse and the replacement of healthcare practitioners. Conclusions: The KELA.AE app was found to be satisfactory and supportive of the participants' needs. Changes were made to the app as suggested during the interviews.

Keywords: Chronic Kidney Failure, Mobile Applications, Health Behavior, Diet, Telemedicine

Submitted: June 24, 2019

Revised: 1st, August 14, 2019; 2nd, September 26, 2019; 3rd, October 27, 2019

Accepted: October 27, 2019

\section{Corresponding Author}

Cosette Fakih El Khoury

Department of Health Services Research, Care and Public Health Research Institute, Maastricht University, Maastricht, Netherlands. Tel: +31-43-388-2446, E-mail: c.fakih@maastrichtuniversity.nl (https://orcid.org/0000-0001-6935-2758)

This is an Open Access article distributed under the terms of the Creative Commons Attribution Non-Commercial License (http://creativecommons.org/licenses/by-nc/4.0/) which permits unrestricted non-commercial use, distribution, and reproduction in any medium, provided the original work is properly cited.

(C) 2019 The Korean Society of Medical Informatics 


\section{Introduction}

A wide range of dietary mobile applications (apps) is readily available and accessible to the public [1,2]. Such apps are mainly used as food and exercise tracking tools $[3,4]$ and their usage, in this context, is effective [2,5]. Accordingly, there is potential for digital interventions because they may reach many persons at a low cost [6].

Most available dietary apps target obesity and diabetes [1,7], and only a few tackle other medical conditions [2]. In some chronic diseases, such as chronic kidney disease (CKD), the complexity of dietary management places a burden on the patient [8]. Patients with CKD are often non-compliant to dietary restriction and particularly to phosphorous restrictions [9]. Lack of knowledge and lifestyle changes, attributed to dietary restrictions, are often the reasons for compliance challenges [9]. Accordingly, hyperphosphatemia is common among hemodialysis patients, and the main characteristic of CKD-mineral bone disorder (CKD-MBD) [9]. Hyperphosphatemia is associated with cardiovascular disease, hyperparathyroidism, and mortality [10]. The central management of phosphorous elevations consists of dietary restrictions and the use of phosphate binders $[11,12]$. Therefore, the management of dietary phosphorous is a particularly challenging task because it includes dietary phosphorous restrictions in parallel to adequate protein intake $[8,13]$. Theory-based interventions and a problem-driven approach may facilitate adherence $[14,15]$. Mobile apps may provide CKD patients with continuous access to self-monitoring and nutrition education $[16,17]$. Commercial mHealth applications for CKD patients are already available on app stores [13]; however, only $50 \%$ of available renal apps reflect evidence-based guidelines [13].

Effective digital interventions, targeting behavioral change, should be iterative, theory-based, and tailored to the needs of users $[18,19]$. To improve acceptability, understanding the perspectives of users, healthcare practitioners, and caregivers is essential in the development process [20]. This is described as a person-based approach [21]. The word 'person', rather than 'user', is used as people that are not necessarily users may influence the usability [21]. Grounding in theories and literature should complement this approach [19].

Several frameworks are available to guide the development of mobile apps; to increase app usability, patient engagement

Table 1. Development of the KELA.AE app based on the Integrate, Design, Assess, Share (IDEAS) framework [20]

\section{IDEAS framework}

Integrate: insights from users and theory

1. EMPATHIZE with target users

2. SPECIFY target behavior

3. GROUND in behavioral theory

\section{Description}

Semi-structured interviews with 6 hemodialysis patients that already own and use smartphone apps. Questions were tailored around:

- Users' experiences with the renal diet

- Their current practices

- Their needs and desires (for support)

Specific target behaviors were extracted based on user feedback and literature. The broad goal identified is 'challenges with adherence to the renal diet'. Specific goals include:

- Restrict phosphorous, potassium, fluids and other dietary components to recommended intakes

- Achieve target blood phosphorous, and potassium values

A meta-analysis was performed to gain an understanding of the effects of the use of dietary mobile applications in chronic diseases on nutritional outcomes in adults.

A multi-behavior theory approach was applied:

- Reasoned Action Approach [27]

- Transtheoretical Model [24]

Theories selection was based on a topic and concept approach (literature in CKD interventions [28], theories used by other dietary apps [2], and concepts identified during brainstorming related to self-efficacy, norms, and attitudes). 
Table 1. Continued 1

\section{IDEAS framework}

Design: iteratively and rapidly with user feedback

4. IDEATE implementation strategies

5. PROTOTYPE potential products

6. GATHER user feedback

7. BUILD minimum viable product

\section{Description}

Brainstorming sessions were held by two of the authors (C.F.K. and M.K.), on multiple sessions, after a revision of available literature and the patients' feedback. Sessions often included additional expert/s:

- Local dietitian

- Communication expert (from the communications department at Zayed University)

- Arabic language expert (from the Arabic department at Zayed University)

- Design team

This process was iterative and main outputs of the sessions were decisions related to:

- Modes of delivery of educational materials (podcasts, videos, notifications, and recipes were developed)

- Content of educational materials and mapping based on theories selected

- Choice of nutrient databases to be included/Arabic food lists categorized as low/medium or high in phosphorous

- Technical matters related to the recording of podcasts and development of videos

- Choice of recipes to be included

- Order of educational messages based on a gradual building of information relayed

Material content was developed in line with KDIGO guidelines [28].

Several steps took place before the development of the first prototype of the app:

- A general wireframe (a document with sketched screenshots of the basic features of the app)

- Detailed wireframe including screenshots of every feature in the app with design and potential graphical outputs

Revision and adjustments of the wireframes continuously took place by C.F.K., M.K. and the design team and continuous changes were applied before the development of a prototype.

Semi-structured interviews were held with patients and healthcare practitioners. The interviewees were shown the app prototype and samples of the educational materials.

- 6 hemodialysis patients that already own and use smartphone apps

- 6 healthcare practitioners (2 nephrologists and 4 dietitians)

An app prototype was developed.

Revision and adjustments of the prototype continuously took place by C.F.K., M.K. and the design team and continuous changes were applied before the development of the final prototype. 
Table 1. Continued 2

\begin{tabular}{l}
\multicolumn{1}{c}{ IDEAS framework } \\
\hline Assess \\
$\begin{array}{ll}\text { 8. PILOT potential efficacy and usability } & \text { This step is in the planning step and will be reported elsewhere. } \\
\text { 9. EVALUATE efficacy in RCT } & \text { This step is in the planning step and will be reported elsewhere. } \\
\text { Share } & \text { The app will be available through app stores to the public and the results of } \\
\text { 10. SHARE intervention and findings } & \text { the research are being and will be submitted for publication at the } \\
& \text { different steps. }\end{array}$ \\
\hline
\end{tabular}

KELA.AE: the Kidney Education for Lifestyle Application to the Internet domain name of the United Arab Emirates, CKD: chronic kidney disease, KDIGO: Kidney Disease Improving Global Outcomes, RCT: randomized controlled trial.

in the design and development process is, therefore, essential [18-21]. The Integration, Design, Assessment, and Sharing (IDEAS) framework suggests a comprehensive stepwise approach that includes a multidisciplinary perspective in the development of a prototype; along with grounding interventions in behavioral theory [20].

Core processes consist of a complex intervention aimed at finding answers to questions raised at various stages within a planning framework. They are essential in the development of theory and evidence-based behavioral change interventions because they present a systematic and implementable way of addressing a problem [15]. Defining problem behavior, brainstorming possible answers, and finding solutions should be included in a stepwise approach [15]. The selection and application of behavioral theories may also be tailored to the topic and the defined problem [15].

This paper aims to describe the person-centered, theorybased development of the Kidney Education for Lifestyle Application (KELA.AE app) for Arabic speaking patients. The .AE was selected as the suffix in the app name to relate the app to the Internet domain name of the United Arab Emirates (UAE).

\section{Methods}

\section{App Development}

The KELA.AE app development followed the IDEAS framework [20] to develop digital health behavior change interventions. The framework proposes a four-step approach, including integration, design, assessment, and sharing; the steps are further broken down into a total of 10 sub-steps [20]. Table 1 illustrates how the development of the KELA. $\mathrm{AE}$ app integrates this framework. The development process was iterative, and the steps were not developed linearly. The development detailed in Table 1 often required a backward flow to a previous step along with amendments and adjustments based on findings in a subsequent step. The development process was extended to more than one year; it started in the fall of 2016, and the first version of the application was available in the spring of 2018. The integration and design steps were completed by the creation of the app prototype, whereas the assessment and sharing steps are still in process (Table 1).

\section{Development of Educational Materials}

Previously validated stage-based Arabic educational materials targeting osteodystrophy [22] were adapted and amended to accommodate the educational modalities of the application and the Emirati dialect. The validated materials are based on the transtheoretical model [23] and are validated both in the English and Arabic languages [22]. The transtheoretical model for behavior and readiness to change proposes a five-stage algorithm: pre-contemplation, contemplation, preparation, action, and maintenance [24]. Despite the problems that exist in the application of the stages proposed by the transtheoretical model [25], stage-based interventions have shown some promising results [24]. The validity of the stages' algorithm proposed by the transtheoretical model is not well established; accordingly, many researchers have changed the existing algorithm [24]. The validated stagebased materials that were used included only three different stages of change (pre-action, action, and maintenance) rather than the five stages proposed by the model [22]. The principal investigators performed a literature review and brainstorming sessions as part of the core processes [15]. Through this process, the authors identified the need to incorporate concepts of self-efficacy, norm, and attitudes. Accordingly, the educational materials were then translated to mobile app features, and the narration was re-created to incorporate additional constructs from the reasoned action approach [26]. 
The Arabic department of a national university in Dubai revised all educational materials. These were then translated back to English to ensure that the information had not been altered.

All information included in the educational and self-monitoring features were in line with the clinical practice guidelines for the diagnosis, evaluation, prevention, and treatment of CKD-MBD of the Kidney Disease Improving Global Outcomes (KDIGO) [27]; thus, the app content was chosen with consideration of evidence-based guidelines.

Three different education modalities were developed based on their reported effectiveness in the literature [28-31]. These include podcasts, videos, and notifications.

Traditional recipes were adapted to the renal diet and were also included in the application. A baseline of Arabic recipes was used from previously validated educational materials [22], and a list of additional typical UAE recipes was collected by a group of local dietetic students from a sample of four UAE nationals' households. Recipes were amended to contain less phosphorous, sodium, and potassium. Local UAE households then prepared and tested the newly developed recipes and confirmed their acceptability.

\section{Interviews, Participants, and Design}

Qualitative, semi-structured interviews with hemodialysis patients and healthcare practitioners (dietitians and nephrologists) were performed. The aim of the interviews was to gather patients and healthcare practitioners' feedback and to involve them in the development process. The interviews with patients assessed the need for an app, the willingness to

Table 2. Sample stage-based podcasts matched to constructs of the reasoned action approach

\begin{tabular}{|c|c|c|}
\hline Stage of change & $\begin{array}{l}\text { Construct from reasoned } \\
\text { action approach }\end{array}$ & Example of podcast script \\
\hline Pre-action & Norm, Capacity/Self-efficacy & $\begin{array}{l}\text { We are going to talk about how small changes can positively affect the } \\
\text { quality of your life. } \\
\text { By following a diet specific to dialysis, exercising, and taking specific } \\
\text { medications, you can improve your lab tests. Your lab tests are } \\
\text { affected by your diet, for example if your diet is high in the mineral } \\
\text { phosphorus, then your phosphorus level in the blood will also rise } \\
\text { and that is not something desired for your health. } \\
\text { I know people, on dialysis, who suffered from itching and sleep } \\
\text { disturbances; the reason for these symptoms was unclear to them, } \\
\text { but by taking care of their diet and lowering the intake of foods high } \\
\text { in the mineral phosphorus, the itching and sleep disturbances im- } \\
\text { proved, and they felt better. You have the power to manage your own } \\
\text { diet, and you will see improvement in your life and reduce the risks } \\
\text { to your health as soon as you make small changes. }\end{array}$ \\
\hline Action & Attitude & $\begin{array}{l}\text { Being on a low phosphorus diet is important to prevent a high blood } \\
\text { phosphorus level, and therefore prevent many complications like } \\
\text { bone or heart diseases. } \\
\text { Phosphorus is present in many foods especially those rich in protein. } \\
\text { That doesn't mean you must cut down on proteins because your body } \\
\text { needs protein for optimal functioning. The solution is to consume } \\
\text { proteins that are as low in phosphorous (P) as possible. } \\
\text { Some examples of high protein foods but low or moderate P are fish, } \\
\text { poultry, lean meats, crab and shrimps. }\end{array}$ \\
\hline Maintenance & Capacity/Self-efficacy & $\begin{array}{l}\text { Make physical activity a habit. This way it will become easier to comply } \\
\text { with. Have fun while exercising, invite your friends, family or } \\
\text { neighbors to join you. } \\
\text { Make an agenda for physical activity to remind you to exercise! After } \\
\text { complying for a while, you will feel very rewarded and proud. }\end{array}$ \\
\hline
\end{tabular}


use an app, and features desired in an app. Interviews with healthcare practitioners included an overview of app features along with assessment of the need for an app by hemodialysis patients.

Patients were recruited at Al Qassimi Hospital Sharjah, UAE. Inclusion criteria were the following: (1) being an adult (aged $\geq 18$ years) hemodialysis patients, (2) owning and being capable of operating any app on a smartphone, (3) being free of life-threatening conditions, (4) having been on hemodialysis for 3 months or longer, and (5) possessing full cognitive capacity. The healthcare practitioners recruited had to be currently practicing in a dialysis unit in the UAE. A prototype of the app, along with sample educational materials, was shown during the interviews. A sample of 6 patients and 6 healthcare practitioners were interviewed. The nature of the study was mainly formative, the scope was narrow, and the topic studied was rather clear [32]. Accordingly, the data gathered was homogenous and sufficient to provide useful information for the app development.

The study was approved by the Institutional Review Board of Zayed University in Dubai (No. MOHAP/DXB/SU BC/ No-5/2016) and informed consent was received from all participants.

\section{Data Collection and Analysis}

Semi-structured interviews were conducted in Arabic by an Emirati local dietitian with each patient and healthcare prac- titioner individually. All interviews were audio-recorded and transcribed verbatim [33] and coded in Arabic using MAXQDA standard version 18.1.1 (1995-2018 VERBI GmbH, Berlin, Germany). Interviews were performed by a moderator (an Emirati dietitian) and an assistant facilitator (C.F.K.). The facilitator conducted each interview while the assistant recorded notes [34]. The qualitative analysis guide of Leuven (QUAGOL) was followed [35]. Stages 1 to 7 (transcription, reading, narrative reports, conceptual schemes, and coding) were performed by two Arabic speaking authors (C.F.K. and D.C.); whereas stages 8 to 10 (analysis of concepts and description of the results) were performed by 3 authors C.F.K., D.C., and M.K.; M.K. also coded two randomly selected transcripts to ensure reliability.

\section{Results}

\section{App Description and Content}

Overall, the app was developed to contain a total of 24 podcasts, three animated videos, and 161 notifications. Videos deliver more complex concepts than the other educational modalities. For example, the videos present concepts such as the phosphorus-to-protein ratio of food and guide patients to identify and consume foods that are rich in proteins yet low in phosphorous. Tables 2, 3, and Figure 1 present some examples of stage-based podcasts, videos, and notifications. Additionally, the app includes 26 recipes along with their

Table 3. Sample stage-based notifications with corresponding reasoned action approach constructs

\begin{tabular}{cll}
\hline $\begin{array}{c}\text { Stage of } \\
\text { change }\end{array}$ & $\begin{array}{c}\text { Construct from reasoned } \\
\text { action approach }\end{array}$ & Sample notification \\
\hline Pre-action & Attitude & Small changes in your daily diet can have a positive impact on your lab tests. \\
& Norm & Having strong bones will allow you to practice fun activities with your family and \\
friends. & Dialysis patients have prevented the occurrence of heart complications by following a \\
& Capacity/Self-efficacy & controlled Phosphorus diet. You can do it too!
\end{tabular}



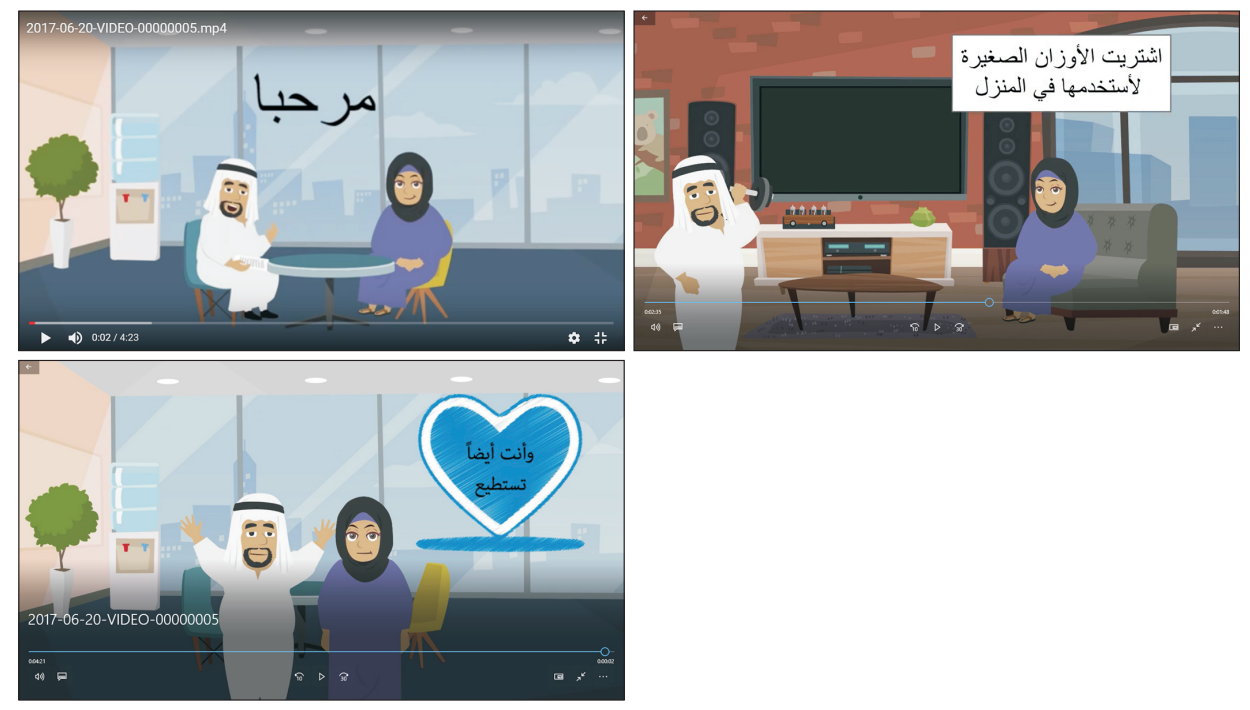

Figure 1. Sample video snapshot.

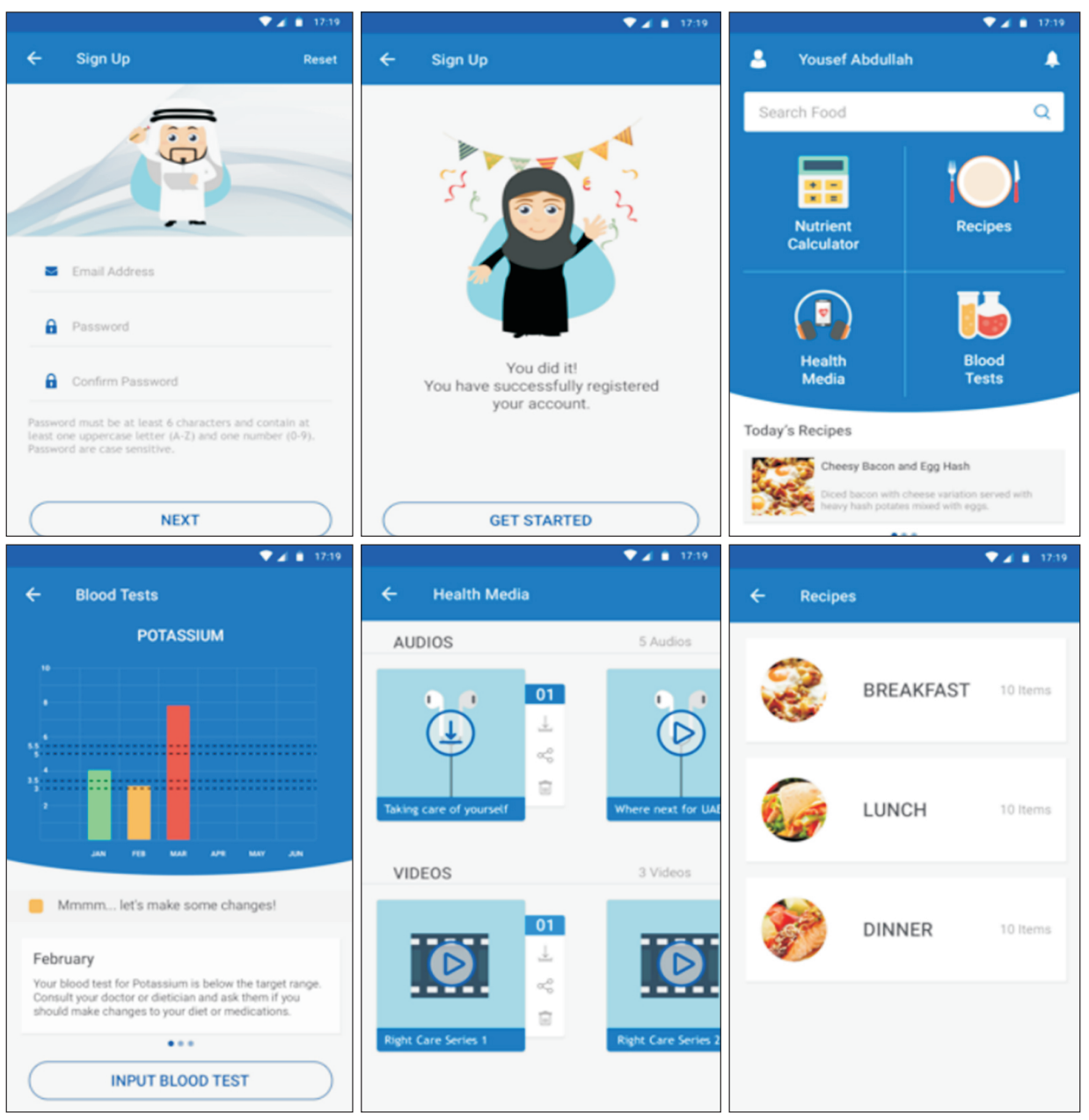

Figure 2. Sample screenshots from the KELA.AE app.

nutrient analysis for salt, potassium, phosphorus, and protein content. Furthermore, users can access self-monitoring features, namely, a food intake diary and a laboratory value tracker. Sample screenshots from the app are presented in Figure 2.
The app name was selected as KELA.AE; 'KELA' stands for Kidney Education for Lifestyle Application, but KELA also means kidney in Arabic. The app design is culture-specific, in terms of dialect, recipes, food selections, and graphics (for example, characters represent male and female Emirati pa- 
tients).

\section{Key Findings from Patients Interviews}

A total of 6 patients were interviewed, 4 males and 2 females. The mean age of the patients was 47 years (range, 37); all patients were of Arab nationalities (4 UAE nationals, one Omani, and one Lebanese) and had been on dialysis for more than 3 months. Though computer literacy levels were not formally measured during development, inclusion criteria addressed the capacity to operate a mobile app. Each interview was started by asking patients about their experience with the renal diet in general. Patients were then shown the app prototype and the educational materials and asked for feedback. Figure 3 summarizes the key findings from the patients' interviews.

\section{1) Experience with the diet}

Frustration concerning access to information was common among patients; many expressed struggles with finding reliable information on the hemodialysis diet. Although dietitians are available at dialysis centers, patients desire continuous access to information concerning food selections. Patients reported using the internet as the most accessible and most convenient platform for answers regarding the diet, despite knowing that it could lack some accuracy. All patients, except one, reported referring directly to their physicians.

They also described their experience in relation to the renal diet as more challenging at the start of dialysis, but they reported that it became more comfortable with time. Moreover, they expressed that sharing information with other dialysis patients facilitated the learning process. Most patients expressed a need for educational features and constant access to useful information through an app rather than selfmonitoring features.

"At the beginning, I was very lost, then I started asking here and there (to understand the diet better), I would see other dialysis patients, and they would talk about certain things, and I would ask, and slowly, slowly I started understanding more" (MP2/71-73).

\section{2) App evaluation}

The patients were shown an app prototype; along with samples of the educational materials. Most of them showed interest in educational materials mainly. Patients expressed a preference for podcasts and videos over traditional paperbased education.

When asked how easy they found the app, on a scale of 1 to 10 , they answered $7 / 10$ as the lowest score, with a median score of $8 / 10$. Some defined the app as 'easy' (FP1/12), or 'very useful' (MP1/24); while one patient was hesitant and preferred being able to use it before providing feedback.

"I would have to access the app and use it to be able to give you any feedback" (MP4/69-70).

Patients were asked to provide possible suggestions or changes to the app during the interview. Two leading recommendations were provided, including a search feature to determine the nutrient content of cultural foods and to have access to the dietitian through the app.

"For example, can the dietitian be on the app so she can send us things?" (MP4/75-76).

\section{Key Findings from Healthcare Practitioner Interviews}

A total of 6 healthcare practitioners were interviewed, in-

- Sub-theme: Current status

- Frustration pertinent lack of access to continuous information

- Constant challenges particularly at the beginning

- Sub-theme: Need for education

- Continuous access

- Education of food selections

- Role of other patients

\section{Theme: App evaluation}

- Sub-theme: Current features

- Preference for educactional features

- How easy is the app on a scale of 1 to 10 ? Median score of $8 / 10$

- Sub-theme: Additional features/suggestions

- Search feature for nutrient composition of cultural foods

- In-app access to the dietitian

Figure 3. Key findings from patients' interviews on the KELA.AE app. 
- Sub-theme: Current features

- Accurate, simple and culturally sensitive

- Concern with app misuse and replacement of healthcare practitioners

- Sub-theme: Additional features/suggestions

- More educational features targeting issues other than CKD-MBD (potassium, diabetes, HTN, fluid management, protein intake, iron...)

Figure 4. Key findings from healthcare professionals on the KEAL. AE app. CKD-MBD: chronic kidney disease-mineral bone disorder, HTN: hypertension. cluding 2 nephrologists and 4dietitians. Healthcare practitioners were shown an overview of all the features of the app, and then they were asked to provide feedback. Figure 4 summarizes the key findings from the interviews with healthcare practitioners.

\section{1) App evaluation}

Overall, healthcare practitioners found the app accurate, simple, and culturally sensitive; the features of the app were considered comprehensive, organized, and tailored to patients' needs. Positive remarks mainly addressed the videos and podcasts, as well as the overall design and graphics of the logo and the application. One nephrologist said: "My feedback is very positive, I like this application, it is very informative, and it will help our patients a lot. Specifically, when it is in both languages, English and Arabic. It is a very positive tool in the management of the patients; because the diet in dialysis patients is a very important aspect of any stage of renal disease patient, and without this, it's very harsh to manage our patients, without this diet component" (NH1/45).

However, some concerns emerged regarding app misuse. One nephrologist expressed fear that patients may exchange information with other dialysis patients to whom such dietary guidelines may not apply.

For instance, one nephrologist said: "if I told one patient that is not diabetic and does not have hypertension if he told his friend which is diabetic and hypertensive, he would take this one and he will... it's not good." (NH2/94).

An additional concern was also discussed, and that is related to the fear of the app replacing healthcare practitioners.

"I would not strongly advise that they should self-estimate their potassium and phosphorus levels every time, they can have an idea, but we would like to advise them to see or consult us that this was our readings in the application so that we can review, or our dialysis dietitian can review them. They can have an idea, but I will not recommend that they solely rely on that." (NH1/51-52).

\section{2) Suggestions}

Healthcare practitioners, including one nephrologist (NH2) and one dietitian (DH1), suggested adding more features, mainly to make the app more comprehensive in terms of coverage for education on potassium, sodium, and fluids as well as diabetes, dyslipidemia, and hypertension management. One nephrologist said: "I would like the recipes to specify if they are suitable for patients with diabetes or hypertension...90\% of dialysis patients suffer from diabetes or hypertension." (NH2/14). Two dietitians suggested including more educational material on protein sources and quantities (DH1 and DH4). Healthcare practitioners (NH2 and DH1) also focused on the need for more information about fluid intake and its management. For example, one dietitian said: "I would suggest adding this part (referring to water), so they can have a part for calculation of such issues (referring to tracking and self-management of fluids)" (DH4/43). Suggestions for more educational material covering additional minerals, such as iron, were made by one nephrologist and one dietitian (NH2 and DH1).

\section{Discussion}

The KELA.AE app was developed based on a personcentered, theory-based approach. The app includes selfmonitoring and educational features. Previous literature has shown that mobile applications may serve as effective selfmonitoring tools [36], and they have the potential to deliver education [13] to patients. Tailoring the app content to patient needs and incorporating behavioral theories are criteria that contribute to well-designed dietary apps [37].

The patients interviewed during the development reported internet usage as a nutrition information source. Such practices have grown in recent years because many people rely on the internet as an information source [38]. A lot of nutritional information is available on the renal diet through websites and YouTube videos [39]. Content analysis showed that most of the information found on websites is evidencebased; however, it is not easily understandable and action- 
able; whereas information on YouTube tended to be less evidence-based yet more understandable [39]. Accordingly, there seems to be a need for information developed based on evidence, addressing the health literacy of renal patients [40].

The results of the interviews with patients emphasized a need for education rather than self-monitoring. These findings have also been described by a recent systematic review, reporting that most available CKD apps contain food dairies and dietary recommendations, whereas CKD-friendly recipes and in-app education were not as common. The review also addressed the need for in-app education to cater to various learning styles [41]. The patients in this study also expressed the need for several educational features; hence, we incorporated recipes and used various education modalities in the KELA.AE app, based on data gathered from interviews and recent literature.

The perspective of other people who may influence app usage is also essential $[42,43]$. Thus, healthcare practitioners were also interviewed and shown an app prototype. Generally, they evaluated the app positively; however, some raised concerns related to app misuse and the replacement of healthcare practitioners.

Digital interventions may support a reciprocal relationship between patients and healthcare practitioners and enhance patient-centered care [44]. However, the extent to which a healthcare practitioner is willing to engage in a collaborative partnership with the patient might influence the effectiveness of eHealth intervention [45]. Additionally, the perceived quality of the physician (defined as perceived physician knowledge and communication skills), and the quality of the information (defined as adequacy, relevance, and usefulness of the information) retrieved by patients online have a significant impact on the patient-physician relationship [46].

Some challenges were faced that were mainly related to technical matters. However, the main limitation was the incorporation of a nutrient database into the self-monitoring feature of the app. Using nutrient data sources developed in different countries may lead to significant errors in the assessment of nutrient composition [47]. Nevertheless, this issue is not only applicable to apps, as practitioners in the Arab world commonly use foreign nutrient databases due to the lack of a reliable Arabic database. Additionally, the database is in the English language, which is a major barrier to Arabic speaking users. Based on patient suggestions, a list of Arabic foods categorized by their mineral content was made available on the app to compensate for this limitation.

The strength of this study lies in the involvement of the patients and healthcare practitioners in app development.
Patients have previously expressed requests to collaborate on the development of solutions tailored to support them in self-management [48]. KELA.AE development involved patients and healthcare practitioners in a structured approach and collected qualitative data that guided the app development. Given the nature of the study, the data gathered was informative; therefore, a larger sample was not required.

Future research should address the effectiveness of CKD apps as educational tools in increasing patient knowledge and adherence to the renal diet.

The KELA.AE app was developed to close the current gap between patients' need for information while incorporating behavioral theories and using an iterative, person-driven approach. We consider this development method supportive for app developers as it is replicable across various cultures, languages, and chronic diseases. Overall, the app review was positive, and patients expressed willingness to use the app, while healthcare practitioners considered it useful. The feasibility, usability, and effectiveness of the app will be assessed through an interventional trial at a later stage.

\section{Conflict of Interest}

No potential conflict of interest relevant to this article was reported.

\section{Acknowledgments}

The app development was funded by Zayed University, United Arab Emirates. We acknowledge the staff and students from Zayed University who volunteered by contributing to the app development at many steps.

\section{ORCID}

Cosette Fakih El Khoury (http://orcid.org/0000-0001-6935-2758)

Mirey Karavetian (http://orcid.org/0000-0001-5916-6740)

Ruud J. G. Halfens (http://orcid.org/0000-0003-2778-9120)

Rik Crutzen (http://orcid.org/0000-0002-3731-6610)

Dayana El Chaar (http://orcid.org/0000-0003-3192-8445)

Jos M. G. A. Schols (http://orcid.org/0000-0002-4062-2061)

\section{References}

1. Martinez-Perez B, de la Torre-Diez I, Lopez-Coronado M. Mobile health applications for the most prevalent conditions by the World Health Organization: review and analysis. J Med Internet Res 2013;15(6):e120. 
2. Fakih El Khoury C, Karavetian M, Halfens RJ, Crutzen R, Khoja L, Schols JM. The effects of dietary mobile apps on nutritional outcomes in adults with chronic diseases: a systematic review and meta-analysis. J Acad Nutr Diet 2019;119(4):626-51.

3. DiFilippo KN, Huang WH, Andrade JE, Chapman-Novakofski KM. The use of mobile apps to improve nutrition outcomes: a systematic literature review. J Telemed Telecare 2015;21(5):243-53.

4. Boyce B. Nutrition apps: opportunities to guide patients and grow your career. J Acad Nutr Diet 2014;114(1):135.

5. Carter MC, Burley VJ, Nykjaer C, Cade JE. 'My Meal Mate' (MMM): validation of the diet measures captured on a smartphone application to facilitate weight loss. $\mathrm{Br}$ J Nutr 2013;109(3):539-46.

6. Bennett GG, Glasgow RE. The delivery of public health interventions via the Internet: actualizing their potential. Annu Rev Public Health 2009;30:273-92.

7. Turner-McGrievy GM, Beets MW, Moore JB, Kaczynski AT, Barr-Anderson DJ, Tate DF. Comparison of traditional versus mobile app self-monitoring of physical activity and dietary intake among overweight adults participating in an mHealth weight loss program. J Am Med Inform Assoc 2013;20(3):513-8.

8. D'Alessandro C, Piccoli GB, Cupisti A. The "phosphorus pyramid": a visual tool for dietary phosphate management in dialysis and CKD patients. BMC Nephrol 2015;16:9.

9. Durose CL, Holdsworth M, Watson V, Przygrodzka F. Knowledge of dietary restrictions and the medical consequences of noncompliance by patients on hemodialysis are not predictive of dietary compliance. J Am Diet Assoc 2004;104(1):35-41.

10. Abe M, Okada K, Soma M. Mineral metabolic abnormalities and mortality in dialysis patients. Nutrients 2013;5(3):1002-23.

11. Melamed ML, Buttar RS, Coco M. CKD-mineral bone disorder in stage 4 and 5 CKD: what we know today? Adv Chronic Kidney Dis 2016;23(4):262-9.

12. Newsome B, Ix JH, Tighiouart H, Sarnak MJ, Levey AS, Beck GJ, et al. Effect of protein restriction on serum and urine phosphate in the modification of diet in renal disease (MDRD) study. Am J Kidney Dis 2013;61(6):10456.

13. Lambert K, Mullan J, Mansfield K, Owen P. Should we recommend renal diet-related apps to our patients? An evaluation of the quality and health literacy demand of renal diet-related mobile applications. J Ren Nutr 2017;27(6):430-8.

14. Clark S, Farrington K, Chilcot J. Nonadherence in dialysis patients: prevalence, measurement, outcome, and psychological determinants. Semin Dial 2014;27(1):429.

15. Ruiter RA, Crutzen R, Kok G. Core processes for developing theory-and evidence-based interventions [Internet]. [place unknown]: PsyArXiv; 2018 [cited at 2019 Oct 25]. Available from: http://doi.org/10.31234/osf.io/ j4ftz.

16. Prest M. Mobile phone applications for kidney patients. J Ren Nutr 2013;23(4):e83-e85.

17. Hutchesson MJ, Rollo ME, Callister R, Collins CE. Selfmonitoring of dietary intake by young women: online food records completed on computer or smartphone are as accurate as paper-based food records but more acceptable. J Acad Nutr Diet 2015;115(1):87-94.

18. Schnall R, Rojas M, Bakken S, Brown W, CarballoDieguez A, Carry M, et al. A user-centered model for designing consumer mobile health (mHealth) applications (apps). J Biomed Inform 2016;60:243-51.

19. Brown W 3rd, Yen PY, Rojas M, Schnall R. Assessment of the Health IT Usability Evaluation Model (HealthITUEM) for evaluating mobile health (mHealth) technology. J Biomed Inform 2013;46(6):1080-7.

20. Mummah SA, Robinson TN, King AC, Gardner CD, Sutton S. IDEAS (Integrate, Design, Assess, and Share): a framework and toolkit of strategies for the development of more effective digital interventions to change health behavior. J Med Internet Res 2016;18(12):e317.

21. Birnbaum F, Lewis D, Rosen RK, Ranney ML. Patient engagement and the design of digital health. Acad Emerg Med 2015;22(6):754-6.

22. Karavetian M, de Vries N, Elzein H, Rizk R, Bechwaty F. Effect of behavioral stage-based nutrition education on management of osteodystrophy among hemodialysis patients, Lebanon. Patient Educ Couns 2015;98(9):111622.

23. Prochaska JO, Velicer WF. The transtheoretical model of health behavior change. Am J Health Promot 1997;12(1):38-48.

24. Brug J, Conner M, Harre N, Kremers S, McKellar S, Whitelaw S. The transtheoretical model and stages of change: a critique: observations by five commentators on the paper by Adams, J. and White, M. (2004) why don't stage-based activity promotion interventions work? Health Educ Res 2005;20(2):244-58. 
25. Adams J, White M. Why don't stage-based activity promotion interventions work? Health Educ Res 2005;20(2):237-43.

26. Fishbein M. A reasoned action approach to health promotion. Med Decis Mak 2008;28(6):834-44.

27. KDIGO clinical practice guideline for the diagnosis, evaluation, prevention, and treatment of Chronic Kidney Disease-Mineral and Bone Disorder (CKD-MBD). Kidney Int Suppl 2009;(113):S1-130.

28. Turner-McGrievy G, Tate D. Tweets, apps, and pods: results of the 6-month mobile pounds off digitally (Mobile POD) randomized weight-loss intervention among adults. J Med Internet Res 2011;13(4):e120.

29. Tuong W, Larsen ER, Armstrong AW. Videos to influence: a systematic review of effectiveness of video-based education in modifying health behaviors. J Behav Med 2014;37(2):218-33.

30. Haapala I, Barengo NC, Biggs S, Surakka L, Manninen P. Weight loss by mobile phone: a 1-year effectiveness study. Public Health Nutr 2009;12(12):2382-91.

31. Kerr DA, Pollard CM, Howat P, Delp EJ, Pickering M, Kerr KR, et al Connecting Health and Technology (CHAT): protocol of a randomized controlled trial to improve nutrition behaviours using mobile devices and tailored text messaging in young adults. BMC Public Health 2012;12:477.

32. Morse JM. Determining sample size. Thousand Oaks (CA): Sage Publications; 2000.

33. Bailey J. First steps in qualitative data analysis: transcribing. Fam Pract 2008;25(2):127-31.

34. Winslow WW, Honein G, Elzubeir MA. Seeking Emirati women's voices: the use of focus groups with an Arab population. Qual Health Res 2002;12(4):566-75.

35. de Casterle BD, Gastmans C, Bryon E, Denier Y. QUAGOL: a guide for qualitative data analysis. Int J Nurs Stud 2012;49(3):360-71.

36. Tanenbaum ML, Bhatt HB, Thomas VA, Wing RR. Use of self-monitoring tools in a clinic sample of adults with type 2 diabetes. Transl Behav Med 2017;7(2):358-63.

37. Azar KM, Lesser LI, Laing BY, Stephens J, Aurora MS, Burke LE, et al. Mobile applications for weight management: theory-based content analysis. Am J Prev Med 2013;45(5):583-9.

38. Pollard CM, Pulker CE, Meng X, Kerr DA, Scott JA.
Who uses the Internet as a source of nutrition and dietary information? An Australian population perspective. J Med Internet Res 2015;17(8):e209.

39. Lambert K, Mullan J, Mansfield K, Koukomous A, Mesiti L. Evaluation of the quality and health literacy demand of online renal diet information. J Hum Nutr Diet 2017;30(5):634-45.

40. Fraser SD, Roderick PJ, Casey M, Taal MW, Yuen HM, Nutbeam D. Prevalence and associations of limited health literacy in chronic kidney disease: a systematic review. Nephrol Dial Transplant 2013;28(1):129-37.

41. Kosa SD, Monize J, D'Souza M, Joshi A, Philip K, Reza S, et al. Nutritional mobile applications for CKD patients: systematic review. Kidney Int Rep 2018;4(3):399-407.

42. Ong SW, Jassal SV, Porter E, Logan AG, Miller JA. Using an electronic self-management tool to support patients with chronic kidney disease (CKD): a CKD clinic selfcare model. Semin Dial 2013;26(2):195-202.

43. Damodaran L. User involvement in the systems design process-a practical guide for users. Behav Inf Technol 1996;15(6):363-77.

44. Macdonald GG, Townsend AF, Adam P, Li LC, Kerr S, McDonald M, et al. eHealth technologies, multimorbidity, and the office visit: qualitative interview study on the perspectives of physicians and nurses. J Med Internet Res 2018;20(1):e31.

45. Ammenwerth E. From eHealth to ePatient: the role of patient portals in fostering patient empowerment. Eur J Biomed Inform 2018;14(2):20-3.

46. Laugesen J, Hassanein K, Yuan Y. The impact of Internet health information on patient compliance: a research model and an empirical study. J Med Internet Res 2015;17(6):e143.

47. Cashel KM, Greenfield H. (1995). The effects of Australian, US and UK food composition tables on estimates of food and nutrient availability in Australia. In: Greenfield H, editor. Quality and accessibility of food-related data: Proceedings of the 1st International Food Data Base Conference. Arlington (VA): AOAC International; 1995. p. 225-40.

48. Rogers D. Patient perspective of smartphone-based apps for CKD self-care. Clin J Am Soc Nephrol 2019;14(4):483-4. 